Meta

Journal des traducteurs

Translators' Journal

\title{
Terminologie et traduction
}

\section{Jean Maillot}

Volume 16, numéro 1-2, mars 1971

Actes du colloque international de linguistique et de traduction.

Montréal, 30 septembre - 3 octobre 1970

URI : https://id.erudit.org/iderudit/003541ar

DOI : https://doi.org/10.7202/003541ar

Aller au sommaire du numéro

Éditeur(s)

Les Presses de l'Université de Montréal

ISSN

0026-0452 (imprimé)

1492-1421 (numérique)

Découvrir la revue

Citer cet article

Maillot, J. (1971). Terminologie et traduction. Meta, 16(1-2), 75-81.

https://doi.org/10.7202/003541ar

Ce document est protégé par la loi sur le droit d'auteur. L'utilisation des services d'Érudit (y compris la reproduction) est assujettie à sa politique d'utilisation que vous pouvez consulter en ligne.

https://apropos.erudit.org/fr/usagers/politique-dutilisation/
Cet article est diffusé et préservé par Érudit.

Érudit est un consortium interuniversitaire sans but lucratif composé de l’Université de Montréal, l'Université Laval et l'Université du Québec à Montréal. Il a pour mission la promotion et la valorisation de la recherche. https://www.erudit.org/fr/ 


\section{Terminologie et traduction}

Je me reporterai pour commencer à près d'un an en arrière, à la réunion qui avait rassemblé professeurs et étudiants de l'École d'interprètes et de traducteurs à laquelle j'apporte mon modeste concours. Au cours de cette réunion, le directeur de l'école avait indiqué, parmi les possibilités de débouchés pour les futurs diplômés, les services de terminologie qui tendent à se multiplier dans les organisations internationales et nationales et dont nul ne songerait à contester l'utilité. Il est certain que les traducteurs sont directement intéressés à disposer de la documentation permettant d'identifier les équivalences entre termes de langues différentes. Il est donc normal qu'ils soient appelés à participer à l'établissement de cette documentation, tout comme la mise au point d'un dispositif technique appelle la coopération des constructeurs et des utilisateurs, quelle que soit la forme sous laquelle elle se réalise, qui peut aller du simple sondage d'opinion aux travaux de normalisation au sein de comités mixtes.

Encore faut-il que les futurs traducteurs soient préparés à cette tâche. Il est donc essentiel qu'ils assimilent les principes de la terminologie, ce qui est précisément l'objet de cet exposé.

Sans vouloir anticiper sur le fait que la structure d'un terme ne peut à elle seule rappeler tous les aspects de la notion qu'il recouvre, je constaterai dès maintenant que le mot terminologie est souvent mal interprété étant donné qu'il fait surtout penser au mot terme. Beaucoup sont enclins à croire qu'il s'agit là d'un synonyme «noble» de vocabulaire. C'est d'ailleurs ce que l'on peut constater dans l'une des recommandations du Comité TC 37 de l'ISO, ayant pour titre «Vocabulaire de la terminologie », ce qui semblerait indiquer à première vue que ces deux mots ne sont pas synonymes, mais qui se contredit en faisant de terminologie un quasi-synonyme de nomenclature, sans toutefois définir ce dernier terme, pas plus que celui de vocabulaire.

Ce n'est qu'à la suite de la définition formulée dans le sens indiqué ci-dessus et donc, très contestable, qu'elle en donne une autre, cette fois plus proche de la vérité. Finalement, on peut définir la terminologie comme la branche des activités linguistiques qui traite des rapports entre les termes et les notions.

Ceci paraît un peu abstrait. En fait, il n'en est rien. Comme dans bien d'autres cas, beaucoup de personnes font de la terminologie sans le savoir : le père ou la mère qui répond à l'enfant l'interrogeant sur le sens d'un terme, 
sous la forme : à quoi ça sert ? comment c'cst fait ? comment ça marche ?, fait de la terminologie en définissant une notion par son aspect utilitaire, constructif, fonctionnel.

De même, en répondant à la question : comment ça s'appelle ? Le ça étant illustré par la mention faite par l'enfant d'un ou de plusieurs des aspects mentionnés ci-dessus, le père ou la mère fait encore de la terminologie, en inversant cette fois le rapport entre le terme et la notion.

Mais il est temps pour moi de revenir au problème que j'ai posé dès le début, celui des rapports entre la traduction et la terminologie, ou encore, si on le place sur le plan des personnes, le problème consistant à déterminer dans quelle mesure un traducteur peut apporter un concours efficace à des travaux de terminologie.

Je pense personnellement que, pour commencer, il est nécessaire que le traducteur en question ait une expérience approfondie de la traduction, ce qui exclut d'emblée les débutants, quel que soit le complément d'instruction qu'ils aient pu recevoir dans le domaine de la terminologie. Il ne suffit pas, non plus, que cette expérience se limite à la simple pratique de la traduction envisagée sous son aspect de la détermination des équivalences entre termes de langues différentes. Si tel est le point particulier sur lequel les travaux de terminologie représentent une aide précieuse pour le traducteur, ce dernier ne doit pas tomber dans l'illusion selon laquelle il suffit d'avoir, au cours d'une carrière bien remplie, enregistré des équivalences permettant de suppléer aux nombreuses erreurs ou lacunes des dictionnaires bilingues ou multilingues d'usage courant, ce qui devrait précisément inciter le traducteur à une certaine prudence quand il entreprend un travail de ce genre, sinon il risque de se trouver dans le même cas que l'un de nos confrères dont le dictionnaire bilingue avait suscité de la part d'une revue scientifique des plus sérieuses la critique suivante :

... l'auteur est persuadé que des erreurs ont pu se glisser dans son ouvrage et il serait très reconnaissant à ceux qui en trouveraient de bien vouloir les lui signaler.

L'ouvrage que nous avons feuilleté présente en fait beaucoup d'erreurs, d'imprécisions et de traductions fantaisistes. Un amateur - ou professionnel - de la «belle ouvrage » trouverait dans les travaux de correction, l'occasion d'écrire un nouveau dictionnaire...

Il en résulte qu'un traducteur isolé sans nulle connaissance des principes mêmes de la terminologie et ne voyant de la traduction que son aspect opératoire, pour ne pas dire mécanique, n'est guère qualifié pour apporter sa pierre à l'édifice commun, même quand il se limite à un travail purement lexicographique.

Si l'on se place maintenant au point de vue des travaux de terminologie proprement dits, il apparaît nettement que ce sont les organisations internationales de normalisation qui sont les mieux placées pour les conduire efficacement. Tout comme la normalisation des produits, des appareils, des machines, a pour objet de faciliter leurs échanges, la normalisation des notions facilite l'échange des idées.

Encore faut-il ici que l'organisation internationale considérée fasse preuve de sa compréhension des problèmes de terminologie, ce qui n'est pas toujours le cas, comme en témoigne une autre recommandation de l'ISO, intitulée "Unification internationale des notions et des termes ». Bien qu'on y trouve parfois de bonnes choses, son titre à lui seul fait apparaître une confusion complète 
entre deux problèmes distincts, celui de l'unification des notions qui est la base même des travaux internationaux et celui de l'unification des termes qui relève la plupart du temps de l'utopie la plus complète, à savoir la fabrication d'une sorte d'esperanto limité au vocabulaire ou l'emprunt massif de termes à une langue déterminée.

La première phase des travaux de terminologie, même si leur objectif final est la détermination des équivalences entre termes, consistera à confronter les notions, sous forme de définitions dans deux langues différentes, ce qui est la seule façon de faire apparaître que deux notions concordent exactement, ou, au contraire, qu'il n'y a pas de concordance possible, ce qui, heureusement, est le cas le plus rare.

Ce travail doit se faire indépendamment du choix du terme correspondant à la notion considérée, ce qui peut paraître à priori illogique, étant donné qu'entre le terme et la notion il doit exister une liaison indissoluble. En fait, si nous reprenons la comparaison entre l'échange des produits et des idées, nous pouvons considérer que la définition de la notion correspond à la spécification du produit et que le terme correspond à l'étiquette qui l'accompagne.

Le terme «étiquette» ne joue ainsi que le rôle d'une référence provisoire dont il convient de vérifier si, dans l'une et l'autre langues, le terme s'applique bien à la notion correspondante, ce qui pose le problème de la confirmation d'un terme existant, parfois du choix entre plusieurs termes possibles, parfois même de la fabrication d'un mot entièrement nouveau, au cas où il manque dans unẹ des langues ou même dans les deux, c'est cc que l'on pourrait exprimer en disant que l'étiquette reste en blanc.

Comme on l'a vu précédemment, un terme ne reproduit pas nécessairement tous les aspects de la notion. Il est même préférable que ce ne soit pas le cas, sans quoi il serait trop long. En poussant les choses à l'extrême on pourrait le considérer comme un simple moyen mnémotechnique ; soit purement conventionnel comme c'est le cas de beaucoup de termes du vocabulaire général, soit, ce qui vaut mieux, empruntant au fonds national de la langue considérée une image qui facilite l'effort de mémoire nécessaire.

Tel est le cas en français du coupe-circuit (à fusible) qui exprime bien la fonction du dispositif qu'il désigne, de même celui de l'anglais circuit-breaker, mais en fait il s'agit de deux dispositifs entièrement différents, coupe-circuit se traduit en anglais par fuse et circuit-breaker en français généralement par disjoncteur. Ceci représente un cas typique de faux amis.

Des exemples de ce genre tendraient à montrer l'avantage que présente l'adoption d'un terme international, ce qui a conduit certains théoriciens à défendre le principe de la «précellence du latin » dont on trouve l'application dans des termes comme transformateur, accumulateur, ne présentant d'une langue à l'autre que de légères différences de terminaison. Encore faut-il remarquer que l'anglais emploiera par exemple pour désigner une batterie d'accumulateurs le terme de storage battery.

Ce principe a d'ailleurs été étendu au grec et on en trouve de nombreux exemples dans le vocabulaire scientifique et plus particulièrement dans le vocabulaire médical où il révèle une nette tendance à l'ésotérisme, ce qui est en 
contradiction flagrante avec l'intérêt qui s'attache à n'employer que des termes intelligibles à tous ceux qui sont amenés à exprimer les notions correspondantes.

On trouve un autre exemple de pareils excès dans la proposition qui a été faite dans un groupe de travail, du domaine électrotechnique cette fois, de baptiser des noms de « relais intranomes» et « relais extranomes 》 les dispositifs connus sous les noms de « relais de mesure» et « relais de tout ou rien», en anglais measuring relay et all or nothing relay respectivement. Ces termes sont très parlants et le passage d'une langue à l'autre ne présente aucune difficulté pour quiconque a une connaissance, même moyenne, des ressources propres de chacune de ces langues.

Je me garderai de m'étendre sur les nombreuses difficultés que présentent ces deux aspects de la terminologie, celui de l'harmonisation des notions, avec les phénomènes d'inversion, de recouvrement incomplet des notions, de la disjonction, etc., puis celui du choix ou de la formation des termes, par emprunt, calque, ou tout autre procédé. Il me suffira de conclure en disant que les deux problèmes doivent être traités successivement, le premier sur le plan international, le second sur le plan national et dans ce dernier cas pour la simple raison que les études correspondantes sont liées entre elles, par les personnes ayant participé aux travaux internationaux ou, plus exactement, par le même groupe de personnes. Il est en effet très rare qu'une personne réunisse au même degré dans le domaine linguistique et le domaine technique (le mot technique étant pris dans son sens le plus large) les connaissances et les aptitudes nécessaires à ce genre d'activité.

C'est précisément faute de satisfaire à toutes ces conditions que tant d'individus ou de comités, se placent aux antipodes de ceux qui font de la terminologie sans le savoir, en croyant faire de la terminologie et en fait en n'en faisant pas du tout.

Parmi les erreurs dont ces groupements sont les plus coutumiers, vient en premier lieu celle qui consiste en une traduction littérale du terme sans chercher à savoir ce qu'il couvre. Autant vaudrait identifier un envoi sans défaire son emballage.

Je citerai pour commencer un exemple emprunté à un bulletin de terminologie d'une organisation européenne dont je me suis empressé d'oublier le nom, d'autant plus qu'elle publie par ailleurs des informations tout à fait valables.

Le bulletin en question proposait de traduire l'abréviation anglaise s.h.p. (shaft horse power) par celle de CVA (chevaux sur l'arbre) ce qui est proprement monstrueux à de multiples points de vue; tout d'abord $\mathrm{CV}$ ne désigne que le cheval fiscal, simple coefficient qui n'a rien d'une unité, le symbole de l'unité dite cheval (ex-vapeur) étant ch et n'ayant d'ailleurs pas la même valeur que le horse power. Mais surtout, dans l'expression anglaise, horse power est pris dans le sens de puissance mécanique, tout comme Britanniques et Américains emploient kVA dans le sens de puissance électrique apparente. Il s'agissait donc dans le cas qui vient d'être cité tout simplement de "puissance sur l'arbre » qui ne nécessite nullement d'ètre abrégé en français. Toutes ces observations dénotent un travail de traducteur inexpérimenté.

Voici un autre exemple, pris cette fois dans les travaux d'un comité fonctionnant uniquement sur le plan français et dont l'appellation comporte le mot « terme » 
et non celui de «terminologie » ce qui est déjà révélateur si on prend ce dernier mot dans son sens véritable. Si j'ajoute que dans sa documentation de propagande il se présente comme le «Bureau d'immigration du vocabulaire», on comprend sans peine l'orientation de son activité.

L'exemple est celui du mot américain kinescope qu'il propose sans plus de " franciser » en cinescope. Remarquons d'emblée que le verbe franciser est impropre, puisque la solution proposée se limite à transcrire le kappa du grec par un $c$ au lieu d'un $k$, il l'est d'autant moins que parmi les termes formés sur la même racine, on trouve bien cinétique et cinématique, mais aussi kinésithérapeute. Mais la faute la plus grave consiste à avoir omis de rechercher le sens du terme américain : construit sur deux racines des plus vagues (mouvement et vue), ce terme désigne à la fois ce que nous appelons d'une part le tube image (de télévision) et de l'autre le vidigraphe (ou vidéo-enregistreur sur film) et que les Britanniques appellent respectivement television picture tube et telerecording equipment, ce qui prouve une fois de plus la supériorité des termes formés à partir des ressources propres de la langue vivante et montre en tout cas le danger de ne pas se reporter aux sources.

De l'emprunt maladroit, je vais revenir à la traduction littérale d'un terme composé de plusieurs mots. C'est l'anglais birth control pour lequel un comité haut placé a recommandé d'employer l'expression de "limitation (ou régulation) des naissances ». Ceci n'est que du mot-à-mot qui révèle de la part des auteurs de cette proposition une certaine renotance de la langue anglaise, dont on sait qu'elle tend à être elliptique dans la formation de ses termes. Si on consulte une personne de langue anglaise sur l'objet de ce control, elle ne vous dira pas que c'est les births, ce qui n'aurait aucun sens, mais le birth rate; il s'agit donc, non pas de naissances, mais de natalité.

Je viens d'ailleurs de consulter le Webster mis à notre disposition à l'entrée de l'amphithéâtre et j'y ai trouvé la définition suivante de birth control : control to limitation of the number of children born, especially by... (suit une liste de détails d'ordre anatomique que je vous épargnerai). Cette définition n'est sans doute pas entièrement satisfaisante, elle a toutefois le mérite de confirmer le caractère quantitatif de cette notion.

Quant à l'anglais control il a fait couler tellement d'encre qu'il est inutile de s'étendre sur le sujet. Si, dans le vocabulaire technique, le français, et aussi beaucoup d'autres langues distinguent nettement «contrôle » de «commande » il est inexact de prétendre que, sur le plan administratif, le mot "contrôle » en français n'implique aucune idée d'autorité. L'argument suivant lequel en France le contrôle ne s'exercerait qu'à posteriori est irrecevable et la vie quotidienne nous apporte de nombreux exemples du contraire. Il en est de même pour les contrôleurs quì tendent de plus en plus à se muer en fonctionnaires d'autorité. Quoi qu'il en soit, les termes de régulation et de limitation sont impropres; le premier du fait qu'il est emprunté au vocabulaire technique où il désigne un procédé de réglage automatique permettant de maintenir constantes ou de faire varier suivant une loi déterminée des valeurs données grâce à la mesure de ces grandeurs mêmes et dont l'équivalent anglais est feed-back control.

Quant à «limitation» il est peut-être moins mauvais, mais tendra à être 
pris dans le langage courant dans le sens d'une limite supérieure, ce qui n'est pas nécessairement le cas. De toute façon, il n'évoque que l'un des aspects liés au control dont l'équivalent français le plus proche en pareil cas n'est autre que « dirigisme».

J'aurais pu très facilement multiplier les exemples. J'ai préféré me limiter à un petit nombre d'entre eux, quitte à les développer davantage, de façon à montrer que l'on ne peut pas traiter de termes indépendamment des notions qu'ils recouvrent.

Le sujet de mon exposé se place ainsi à la charnière des deux thèmes traitant respectivement de la traduction spécialisée et de questions relatives à la banque de mots. Si le but recherché consiste à déterminer les équivalents de termes étrangers, il est essentiel de passer par l'intermédiaire de leur définition.

J'avouerai humblement que mon expérience en matière de banque de mots est très limitée. Toutefois, l'un de ces «banquiers », apprenant que je m'occupais, non seulement de traduction, mais aussi de terminologie, m'avait proposé d'être son «client». En le remerciant de son aimable invitation, je lui avais dit que je me voyais plutôt dans le rôle du fournisseur, ayant le privilège d'appartenir à une organisation qui pratique la terminologie depuis près d'un demi-siècle et par conséquent plus qualifiée que les comités ne disposant pas d'une expérience comparable et qui l'approvisionnent en termes établis selon des critères souvent discutables.

Le terme de banque nous fait penser à celui de monnaie ; sans aller jusqu'à parler de faux-monnayeurs, il est certain que les éléments dont sera constituée une banque de mots seront de valeur très inégale suivant leur origine.

Je reviendrai une fois de plus, mais ce sera la dernière, sur la comparaison entre les normes et les produits industriels. Pour ces derniers, il existe une procédure d'homologation garantissant la conformité à une norme, notamment en ce qui concerne la sécurité qu'ils offrent à leurs utilisateurs. Tel est l'objet des marques qui caractérisent l'approbation d'un produit par une organisation telle que l'Association canadienne de normalisation, pour ne citer que le pays qui nous a si aimablement invité.

Il en est de même pour les termes d'une banque de mots, leur emploi pose aussi un problème de sécurité. Il me paraît donc nécessaire de leur attribuer au même titre une sorte de marque de conformité sous la forme d'une simple référence à la source dont ils sont extraits. Je pense qu'il s'agit là d'une précaution élémentaire ayant pour objet de donner à l'utilisateur une notion exacte du degré de fiabilité que présentent des éléments d'origines très diverses.

Pour conclure sur une note moins austère et pour bien montrer que la terminologie est loin d'être une science inaccessible rappelons que les enfants font de la terminologie sans le savoir. Ils ne sont pas les seuls et certains psychologues en ont fait autant, précisément à propos de l'enfance, comme en témoigne un ouvrage dont j'ai eu connaissance tout à fait fortuitement, celui d'Alfred Binet et Th. Simon, intitulé : la Mesure du développement de l'intelligence chez les jeunes enfants.

Parmi les tests proposés par les auteurs, il s'en trouve qui sont précisément du domaine de la terminologie, étant donné qu'ils consistent à demander à l'enfant 
la définition d'un mot déterminé. Les constatations qu'ils ont faites sont, comme vous allez le voir, très originales : dès 6 ans, les réponses révèlent un état d'âme exclusivement utilitaire. À la question demandant «qu'est-ce que c'est qu'un cheval», le sujet interrogé répondra dans son langage enfantin : «c'est pour traîner les voitures, c'est pour courir ». Une table? «c'est pour manger, c'est où qu'on met les assiettes». Une maman? «c'est pour embrasser, c'est pour soigner les petits enfants $\gg$.

Par la suite, l'emploi d'expressions telles que : c'est un animal, c'est un meuble, c'est une dame, dénote des définitions moins enfantines que les précédentes et montre surtout que le sujet interrogé rattache la notion considérée à un ensemble par la référence à un terme générique, ce qui est également un point capital en matière de terminologie.

Dans d'autres cas, l'enfant s'essaie à une description : une fourchette est une petite fourche avec quatre pointes. On retrouve ici l'aspect constructif mentionné précédemment. De même des enfants plus âgés s'occupent de la nature ou de la composition de l'objet de la question : la fourchette est en métal, le cheval, c'est de la viande. Ceci représente encore une variante de l'aspect constructif, bien que dans le second cas on puisse aussi penser à l'aspect utilitaire si l'enfant a pour parents les clients d'une boucherie chevaline.

Les citations qui précèdent ne donnent qu'une faible idée de la variété des réponses enregistrées par ces éminents psychologues. Elles ont toutefois le mérite d'éclairer d'un jour nouveau les divers aspects de la question et de consacrer ainsi le caractère universel de la terminologie.

JEAN MaILlot

\section{QUESTIONS}

M. Dubuc : D'abord, je voudrais souligner deux points qui me semblent très importants dans l'exposé de M. Maillot. Le premier, c'est de faire en quelque sorte de la définition la pierre angulaire de la recherche terminologique. Le second me semble également important : c'est l'identification de la source. C'est une mesure indispensable si l'on veut vraiment justifier les équivalents proposés. Il est toutefois une question que j'ai déjia abordée vaguement et sur laquelle je voudrais revenir de façon peut-être un peu plus explicite. II s'agit de la nécessité de cesser de a se chicaner » entre organismes de recherches terminologiques pour imposer une solution. Je pense qu'il faut rationaliser notre recherche terminologique et surtout avoir l'humilité d'accepter que d'autres que nous puissent faire quelque chose de bien.

Réponse : Dans les questions de terminologie chacun peut apporter sa pierre à l'édifice commun, c'est ce que je voulais dire en parlant du certificat d'origine lorsque j'ai parlé de la Commission électro-technique internationale. Mais il y a également et spécialement, en matière de télécommunications un autre organisme qui s'occupe de terminologie et c'est l'Union internationale des télécommunications, dont les définitions et termes ont peut-être une valeur supérieure à celle d'organismes de normalisation qui ne sont pas gouvernementaux, étant donné que l'Union internationale des télécommunications est une organisation intergouvernementale et que toutes les publications qu'elle produit ont valeur légale dans les pays membres. Je considère toutefois que sur le plan national la meilleure source, et la source la plus sûre de documentation en matière de terminologie, sont les normes. Evidemment elles ont un certain défaut, les travaux de terminologie scientifique et technique dans le cas de la normalisation sont extrêmement longs. On passe des mois et des mois à polir des définitions, à chercher à discerner quel est le terme qui convient et puis le temps passe. Pendant ce temps, les mauvaises habitudes s'installent et il est très difficile de réagir. 